\title{
ANÁLISE BIBLIOMÉTRICA DA PRODUÇÃO CIENTÍFICA DA REVISTA DE ADMINSTRAÇÃO DA UFSM: EM BUSCA DE NOVAS PERSPECTIVAS E DESAFIOS
}

\author{
BIBLIOMETRIC ANALYSIS OF SCIENTIFIC \\ PRODUCTION IN REVISTA DE ADMINISTRAÇÃO DA \\ UFSM: IN SEARCH OF NEW \\ PERSPECTIVES AND CHALLENGES
}

\author{
Recebido 08/04/2013 \\ Aceito 14/06/2013 \\ Jordana Marques Kneipp ${ }^{1}$ \\ Luciana Aparecida Barbieri da Rosa² \\ Roberto Schoproni Bichueti ${ }^{3}$ \\ Ranice Höehr Pedrazzi Pozzer ${ }^{4}$ \\ Vitor Francisco Schuch Júnior ${ }^{5}$
}

\section{RESUMO}

A Revista de Administração da Universidade Federal de Santa Maria - ReA/UFSM, criada em 2008, é uma publicação online do Departamento de Ciências Administrativas do Centro de Ciências Sociais e Humanas da UFSM. A ReA/UFSM vem conquistando o seu espaço no meio acadêmico como um importante veículo para difusão das publicações na área de Administração. Nesse sentido, este estudo teve como objetivo analisar as publicações científicas daReA/UFSM no período de 2008 a 2011. Para tanto, foram analisados todos os artigos publicados na revista nos últimos quatro anos, o que representa 113 artigos. Dentre os principais resultados, constatou-se que os artigos analisados são em sua maioria empíricos e de natureza exploratória, predominando a abordagem e os métodos qualitativos. Os dados coletados e analisados permitem traçar um panorama das publicações da ReA/UFSM no período de 2008 a 2011, possibilitando que a partir dos resultados sejam definidas novas perspectivas e desafios para a revista.

Palavras-chave: Produção Científica, Análise Bibliométrica, Revista de Administração da UFSM.

\footnotetext{
1 Possui graduação em Administração pela Universidade Federal de Pelotas - UFPel e mestrado em Administração pela Universidade Federal de Santa Maria - UFSM. Atualmente é doutoranda no Programa de Pós-Graduação da Universidade Federal de Santa Maria e bolsista da Coordenação de Aperfeiçoamento do Pessoal de Nível Superior (CAPES). Santa Maria, Rio Grande do Sul, Brasil. E-mail: jordanakneipp@yahoo.com.br.

${ }^{2}$ Possui graduação em Administração pela Faculdade de Educação São Luis - FESL, especialização e em Educação Ambiental e mestrado em Administração pela Universidade Federal de Santa Maria - UFSM. Atualmente é professora substituta do Departamento de Ciências Administrativas e aluna do Programa Especial de Graduação de Formação de Professores para a Educação Profissional - PEG. Santa Maria, Rio Grande do Sul, Brasil. E-mail: lucianaaparecidabarbieri@yahoo.com.br.

${ }^{3}$ Possui graduação e mestrado em Administração pela Universidade Federal de Santa Maria - UFSM. Atualmente é Professor Substituto do Departamento de Ciências Administrativas - UFSM e Doutorando do Programa de Pós Graduação em Administração PPGA/ UFSM - Bolsista da Coordenação de Aperfeiçoamento do Pessoal de Nível Superior / CAPES. Santa Maria, Rio Grande do Sul, Brasil. E-mail: robertobichueti@hotmail.com.

${ }^{4}$ Possui graduação em Comunicação Social - Jornalismo, especialização em Gestão da Qualidade e mestrado em Administração pela Universidade Federal de Santa Maria - UFSM. Santa Maria, Rio Grande do Sul, Brasil. E-mail: ranicepedrazzi@hotmail.com.

${ }^{5}$ Possui graduação em Administração e Pedagogia pela Universidade Federal de Santa Maria - UFSM, mestrado em Administração pela Universidade Federal do Rio Grande do Sul - UFRGS e doutorado em Educação pela Universidade Estadual de Campinas - UNICAMP. Atualmente é Professor Colaborador Voluntário no curso de graduação em Administração na Universidade Federal de Santa Maria - UFSM.
} 


\begin{abstract}
The Revista de Administração da Universidade Federal de Santa Maria - ReA/UFSM, founded in 2008, is an online publication of the Departamento de Ciências Administrativas of Centro de Ciências Sociais e Humanas from UFSM. The ReA/UFSM is gaining its place in academiaas an important vehicle for dissemination of publications in the business area. In this sense, this study aimed at analyzing the scientific publications of ReA/UFSM, in the period from 2008 to 2011. Thus, we analyzed all papers published in the periodic, in the last four years, representing 113 studies. Among the main results, it was found that the papers analyzed are mostly empirical, exploratory in nature, with qualitative approach predominantly. The analyzed data allow present an overview of the publications of ReA/UFSM, in the period from 2008 to 2011, enabling to defined new perspectives and challenges for the periodic.
\end{abstract}

Keywords: Scientific Production, Bibliometrics Analysis, Revista de Administração da UFSM.

\title{
1 INTRODUÇÃO
}

Os periódicos científicos possuem um papel fundamental na consolidação de determinada área do conhecimento, tendo em vista que são meios para a divulgação e a difusão dos resultados de pesquisas acadêmicas. Segundo Cunha (1997) o periódico consiste em um espaço para a divulgação e o registro dos resultados de pesquisas empíricas e teóricas e visa promover a integração entre acadêmicos que possuem interesses afins, de forma a criar as denominadas comunidades científicas.

$\mathrm{Na}$ área de Administração no Brasil é crescente o número de veículos para a divulgação da produção acadêmica. Para Wood Jr. eChueke (2008), o crescimento do número de eventos e periódicos na área de Administração no Brasil possui como um dos principais objetivos absorver a produção científica local, tendo em vista que as publicações tornaram-se foco permanente de atenção e preocupação dos pesquisadores e de suas instituições.

Desse modo, tendo em vista o caráter essencial das publicações na atividade dos pesquisadores e a necessidade de preservação dos periódicos brasileiros, que enfrentam inúmeros desafios para garantir a sua longevidade, o presente estudo possui como objetivo analisar as publicações científicas da Revista de Administração da Universidade Federal de Santa Maria - ReA/ UFSM no período de 2008 a 2011. A ReA/UFSM, criada em 2008, é uma publicação online do Departamento de Ciências Administrativas do Centro de Ciências Sociais e Humanas da UFSM. A Revista possui periodicidade quadrimestral e em quatro anos de atuação publicou 113 artigos, buscando construir e ampliar a teoria administrativa e contribuir com a prática de gestão.

A análise da produção científica de um periódico da área de Administração que iniciou as suas atividades há poucos anos, torna-se relevante pelo fato de possibilitar traçar um panorama das publicações e da evolução do periódico, bem como estabelecer novas perspectivas e desafios a fim de garantir a consolidação e a longevidade da revista.

Este estudo está estruturado da seguinte maneira: inicialmente, será apresentada uma seção que trata da importância dos periódicos científicos e traz um breve histórico da ReA/UFSM. Em seguida, evidencia-se o método utilizado para o desenvolvimento do estudo, a análise e discussão dos resultados encontrados e, por fim, as considerações finais. 


\section{A IMPORTÂNCIA DOS PERIÓDICOS CIENTÍFICOS E A REVISTA DE ADMINISTRAÇÃO DA UNIVERSIDADE FE- DERAL DE SANTA MARIA}

A produção científica e a sua respectiva publicação possui caráter fundamental no que se refere às atividades dos programas de pós-graduação (WOOD JR. e CHUEKE, 2008). Alguns veículos, tais como eventos e periódicos científicos são fundamentais para a divulgação e o compartilhamento do conhecimento em determinada comunidade científica.

Nesse sentido, Wood Jr. e Chueke (2008) ressaltam o crescimento em número de eventos e de novas revistas científicas na área de Administração no Brasil, o que evidencia a considerável evolução da área nas últimas décadas. Para os autores, o crescimento do número de eventos e periódicos possui como um dos principais objetivos absorver a produção científica local, tendo em vista que as publicações tornaram-se foco permanente de atenção e preocupação dos pesquisadores e de suas instituições. Complementando Serra, Fiates e Ferreira (2008) ressaltam que pesquisadores em todo o mundo enfrentam o desafio de publishorperish, já que a avaliação da produtividade de um pesquisador está diretamente relacionada às suas publicações de artigos em revistas indexadas e bem qualificadas.

Por outro lado, Bertero (2011) chama a atenção para a não longevidade dos periódicos, já que não é pequeno o número de periódicos que inicia suas atividades, depois atrasa as publicações e acaba por reduzir a sua frequência até o seu encerramento. O autor (2011) ainda coloca que os desafios são ainda maiores para periódicos científicos de Administração brasileiros, já que a área é tradicionalmente caracterizada pela maior ênfase à prática e à divulgação de bestpractices em detrimento à indagação e à problematização; e ainda pelo fato de que no Brasil a vida universitária começou com atraso, e a Administração não fez parte dos primeiros cursos superiores do país.

A análise da produção científica de um periódico permite vislumbrar a sua evolução, identificar lacunas e definir perspectivas. No Brasil, de acordo com Jabbour, Santos e Barbieri (2008), a prática de publicação de balanços científicos da produção acadêmica em Administração começou a ter maior ênfase a partir da década de 1990. O autor afirma que a análise da produção científica na área de ciências humanas, abordando as subáreas de economia e administração, são destaques em periódicos internacionais.

Segundo com Jabbour, Santos e Barbieri (2008), os primeiros estudos desse tipo eram focados em analisar a produção científica concernente ao tema organizações, sendo seguidos por estudos em diversas subáreas da administração, tais como: estudos de procedimentos metodológicos de artigos em estratégia e; produção científica do terceiro setor. Esses estudos identificaram as metodologias mais empregadas e as lacunas teóricas e práticas que poderiam ser exploradas, contribuindo para o avanço da pesquisa científica naquelas áreas.

Nesse contexto, insere-se a ReA/UFSM, um periódico criado em 2008 e que pertence a uma Instituição de Ensino Superior (IES) do interior do estado do Rio Grande do Sul com mais de 50 anos de tradição e considerada uma das mais importantes Universidades do país em termos de publicações de impacto internacional. A idealização da publicação da ReA/ UFSM decorreu de um processo de amadurecimento da atividade docente de graduação e de pós-graduação do Departamento de Ciências Administrativas do Centro de Ciências Sociais e Humanas da UFSM. Atuando no ensino em nível de graduação desde 1967, em nível de Pós-Graduação Lato Sensu desde 1995 e em nível Stricto Sensu desde 2003, o Departamento de Ciências Administrativas assumiu o compromisso com o desenvolvimento acadêmico e empresarial local, regional e na- 
cional. Desse modo, a ReA nasce como uma proposta de fortalecimento e de consolidação das atividades acadêmicas desenvolvidas, tendo como base a credibilidade institucional da UFSM.

A Revista consiste em uma publicação periódica, quadrimestral, aberta a todos os temas e ensaios, teóricos e empíricos, de origem nacional e internacional, da área de Administração, que visem a promover o debate acadêmico e a apresentar práticas de gestão relevantes e aplicadas à realidade organizacional. O periódico desde a sua criação está totalmente on-linee pode ser acessada no endereço: http://www.ufsm.br/reaufsm. Em 2009 a Revista passou a ser elaborada na base do Sistema Eletrônico de Editoração de Revistas (SEER), o que proporcionou o melhor gerenciamento do processo editorial e a otimização dos esforços da equipe, permitindo um maior controle, a organização e a gestão integrada de todo o processo de editoração, com papel fundamental para o sucesso do periódico.

A Revista de Administração da UFSM está classificada no sistema Qualis da Coordenação de Aperfeiçoamento de Pessoal de Nível Superior (CAPES) como B3 na área de Administração, Ciências Contábeis e Turismo e B4 nas áreas de Psicologia, Interdisciplinar e Engenharias III. Em 2010 a revista foi indexada ao Directoryof Open Access Journals (DOAJ) e ao Sistema regional de informaciónen línea para revistas científicas de América Latina, el Caribe, España y Portugal (Latindex) e em 2011 foi indexada à Red de Revistas Científicas de América Latina y el Caribe, España Y Portugal (Redalyc) e ao Sumário de Revistas Brasileiras (Sumários.org).

A ReA/UFSM representa um mecanismo difusor do conhecimento científico na área de Administração e apesar de ter sido criada há menos de 5 anos, a revista vem buscando a sua consolidação como um importante e renomado periódico da área. A recente qualificação no sistema Qualis/CAPES como B3 na área de Administração e as indexações em renomadas bases, denotam a qualidade e a representatividade do periódico no meio acadêmico.

\section{MÉTODO DO ESTUDO}

O presente estudo teve como objetivo analisar as publicações científicas da ReA/UFSM no período de 2008 e 2011.A pesquisa caracteriza-se como quantitativa e foi conduzida por meio de uma pesquisa bibliométrica. Segundo Silva (2004), a bibliometria possui como objetivo analisar a atividade científica ou técnica através do estudo quantitativo das publicações. Foram analisados todos os artigos publicados nos quatro últimos volumes da ReA/UFSM, o que representa 113 artigos.

\subsection{Modelo conceitual}

As variáveis analisadas neste estudo foram definidas a partir da pesquisa de Pinto e Lara (2008) ao analisarem artigos publicados sobre o tema comportamento do consumidor, nos principais veículos de divulgação da produção acadêmica em marketing no Brasil no período de 1997 a 2006. Pinto e Lara (2008) utilizaram um modelo conceitual desenvolvido com base em estudos anteriores de Hoppen, Moreau e Lapointe (1997), Perin et al. (2000) e Gonçalves e Meirelles (2004). Dessa forma, a partir da adaptação do modelo conceitual proposto por Pinto e Lara (2008), foram obtidas as variáveis para proceder a análise bibliométrica, conforme dispostas no Quadro 1. 


\begin{tabular}{|c|c|c|}
\hline & VARIÁVEIS ANALISADAS & CRITÉRIOS \\
\hline \multirow{3}{*}{$\begin{array}{l}\text { Características } \\
\text { gerais das publi- } \\
\text { cações }\end{array}$} & Artigos publicados por ano & Quantidade de publicações \\
\hline & Instituições de Ensino & Instituições dos autores \\
\hline & Temáticas Abordadas & $\begin{array}{l}\text { Classificação de acordo com as subáreas da Adminis- } \\
\text { tração segundo a classificação da CAPES }\end{array}$ \\
\hline \multirow{3}{*}{$\begin{array}{l}\text { Embasamento } \\
\text { conceitual }\end{array}$} & Teorias e conceitos de base & Aprofundado, superficial ou ausente \\
\hline & Objetivos & Sim ou não \\
\hline & $\begin{array}{l}\text { Questão de pesquisa, hipó- } \\
\text { teses ou pressupostos de } \\
\text { base }\end{array}$ & Sim ou não \\
\hline \multirow{5}{*}{$\begin{array}{l}\text { Aspectos meto- } \\
\text { dológicos das } \\
\text { publicações }\end{array}$} & Tipo de artigo & Empírico ou teórico \\
\hline & Abordagem da pesquisa & Qualitativa, quantitativa ou qualitativa-quantitativa \\
\hline & Natureza da pesquisa & Exploratória, descritiva ou causal \\
\hline & Método & $\begin{array}{l}\text { Survey, experimentos, base qualitativa ou bibliomé- } \\
\text { trico. }\end{array}$ \\
\hline & Desenho de pesquisa & Modelo de pesquisa, operacionalização das variáveis \\
\hline \multirow[b]{2}{*}{ Coleta de dados } & Tipo de dados & Primários, secundários ou ambos \\
\hline & Forma de coleta de dados & $\begin{array}{l}\text { Survey e experimento: entrevista pessoal, correios, } \\
\text { eletrônico telefone ou outro; } \\
\text { Base qualitativa: pesquisa documental, entrevista não } \\
\text { estruturada, entrevista estruturada, entrevista semi- } \\
\text { estruturada, técnicas projetivas, grupos de foco ou } \\
\text { outros; } \\
\text { Bibliométricos: pesquisa bibliométrica em publica- } \\
\text { ções. }\end{array}$ \\
\hline \multirow{3}{*}{$\begin{array}{l}\text { Análise dos } \\
\text { dados }\end{array}$} & Confiabilidade & Ausente, Cronbach ou outro \\
\hline & Validade & Ausente, convergente/ discriminante ou outro \\
\hline & Tipo de análise & $\begin{array}{l}\text { Survey, experimento e bibliométrico: univariada, biva- } \\
\text { riada, multivariada ou outros. } \\
\text { Base qualitativa: análise de conteúdo, análise do dis- } \\
\text { curso, análise de significado ou outros. }\end{array}$ \\
\hline \multirow{2}{*}{$\begin{array}{l}\text { Limitações e re- } \\
\text { comendações }\end{array}$} & Limitações do estudo & Sim ou não \\
\hline & $\begin{array}{l}\text { Recomendações para pes- } \\
\text { quisas futuras }\end{array}$ & Sim ou não \\
\hline
\end{tabular}

Quadro 1 - Modelo conceitual para a análise bibliométrica

Fonte: Adaptado de Pinto e Lara (2008)

A análise bibliométrica foi realizada em duas etapas, de modo que inicialmente foram analisadas as características gerais, o embasamento conceitual e os aspectos metodológicos dos 113 artigos analisados. A seguir, procedeu-se a análise de acordo com o método utilizado: survey, base qualitativa, bibliométrico e experimento, sendo que, de acordo com as particularidades de cada método, foram destacados os seguintes aspectos: natureza da pesquisa, embasamento conceitual, coleta e análise dos dados e limitações e recomendações.

Para fins de análise dos dados foram utilizados os softwares SPSS e Excel.

\section{APRESENTAÇÃO E ANÁLISE DOS RESULTADOS}

A seguir são apresentados os principais resultados do estudo, no que se refere à produção científica da ReA/UFSM. Inicialmente apresentam-se as características gerais das publicações e por fim os seus aspectos metodológicos. 
4.1 Análise das características gerais dos artigos publicados naReA/UFSM

No que se refere às características gerais das publicações foram analisados os seguintes aspectos: ano de publicação, instituições, temáticas abordadas nas publicações.

\subsubsection{Análise das publicações por ano}

A Tabela 1 apresenta o número de artigos publicados por ano na Revista de Administração.

Tabela 1 - Artigo publicados por ano

\begin{tabular}{cc}
\hline ANO & QUANTIDADE DE ARTIGOS \\
\hline 2008 & 28 \\
2009 & 29 \\
2010 & 29 \\
2011 & 27 \\
\hline Total & 113 \\
\hline
\end{tabular}

No período de 2008 a 2011 constatou-se que o número de artigos publicados por ano é uniforme, sendo em média 28 artigos.

\subsubsection{Principais instituições das publicações daReA/UFSM}

Com base nas informações declaradas pelos autores a respeito das instituições as quais estão vinculados, foi possível identificar aquelas que mais se destacaram nas publicações no periódico. A Tabela 2 apresenta as principais instituições que publicaram na ReA/UFSM.

Tabela 2 - Principais instituições que publicaram na ReA/UFSM

\begin{tabular}{l|c}
\hline \multicolumn{1}{c}{ INSTITUIÇÕES DE ENSINO } & AUTORES \\
\hline Universidade de São Paulo & 35 \\
\hline Universidade Federal de Santa Maria & 35 \\
\hline Universidade Federal de Minas Gerais & 13 \\
\hline Universidade Federal do Rio Grande do Sul & 9 \\
\hline Universidade FUMEC & 8 \\
\hline Pontifícia Universidade Católica do Paraná & 8 \\
\hline Universidade Federal de Lavras & 7 \\
\hline Universidade Federal de Pernambuco & 6 \\
\hline Pontifícia Universidade Católica de São Paulo & 5 \\
\hline Universidade Federal de Santa Catarina & 5 \\
\hline Universidade Federal de Viçosa & 4 \\
\hline Universidade Estadual de Maringá & 4 \\
\hline Universidade Estadual de Londrina & 4 \\
\hline Universidade Federal de Uberlândia & 4 \\
\hline Universidade Metodista de Piracicaba & 4 \\
\hline Universidade de Passo Fundo & 113 \\
\hline Outras 75 Instituições & $275^{*}$ \\
\hline Total & \\
\hline * O número de instituições é maior que o número de artigos publicados no periódico por se referir à instituição de \\
origem do pesquisador e ao fato de alguns artigos contarem mais de um pesquisador entre os autores.
\end{tabular}


Os resultados obtidos demonstram que as instituições que obtiveram um maior número de autores que publicaram na ReA/UFSM foram a Universidade de São Paulo, a Universidade Federal de Santa Maria e a Universidade Federal de Minas Gerais.

\subsubsection{Temáticas abordadas}

Os artigos publicados na ReA/UFSM foram classificados de acordo com as subáreas da Administração segundo a classificação da Coordenação de Aperfeiçoamento de Pessoal de Nível Superior (CAPES), conforme apresentado na Tabela 3.

Tabela 3 - Área do Conhecimento em Administração

\begin{tabular}{l|c}
\multicolumn{1}{c}{ Área do conhecimento em administração } & Artigos \\
\hline Administração de Empresas & 50 \\
\hline Administração de Recursos Humanos & 21 \\
\hline Mercadologia & 20 \\
\hline Administração de Produção & 7 \\
\hline Administração de Setores Específicos & 4 \\
\hline Administração Financeira & 4 \\
\hline Administração Pública & 4 \\
\hline Negócios Internacionais & 3 \\
\hline
\end{tabular}

Foi possível verificar que as publicações da ReA/UFSM enquadram-se predominantemente nas áreas de Administração de Empresas, Administração de Recursos Humanos e Mercadologia.

\subsection{Análise do embasamento conceitual dos artigos publicados na ReA/UFSM}

A Tabela 4 apresenta as características analisadas no que se refere ao embasamento conceitual dos artigos, a saber: teorias e conceitos de base, objetivos e questão de pesquisa, hipóteses ou pressupostos de base.

Tabela 4 - Embasamento conceitual dos artigos

\begin{tabular}{lccc}
\hline Embasamento Conceitual & & $\mathbf{N}$ & \% \\
\hline Teorias e conceitos de base & Aprofundado & 51 & $45 \%$ \\
\cline { 2 - 4 } & Superficial & 60 & $53 \%$ \\
\cline { 2 - 4 } & Ausente & 2 & $2 \%$ \\
\hline Objetivos & Sim & 105 & $93 \%$ \\
\hline Questão de pesquisa, hipóteses ou pressuposto & Não & 8 & $7 \%$ \\
\cline { 2 - 4 } de base & Sim & 46 & $41 \%$ \\
\hline Total & Não & 67 & $59 \%$ \\
\hline
\end{tabular}

Os resultados da pesquisa demonstram a predominância de teorias e conceitos de base superficiais e que a maior parte dos artigos apresenta objetivos. Contudo a maioria dos estudos não apresenta questão de pesquisa, hipótese ou pressuposto de base. 
4.3 Análise dos aspectos metodológicos dos artigos publicados na ReA/UFSM

A seguir serão apresentados os aspectos metodológicos das publicações: tipo de artigo, abordagem de pesquisa, método utilizado, natureza de pesquisa, embasamento conceitual.

\subsubsection{Tipo de artigo}

A Tabela 5 apresenta a distribuição dos artigos quanto ao tipo.

Tabela5 - Classificação dos artigos quanto ao tipo

\begin{tabular}{lcc}
\hline Tipo de artigo & $\mathbf{N}$ & $\mathbf{\%}$ \\
\hline Empírico & 86 & $76 \%$ \\
\hline Teórico & 27 & $24 \%$ \\
\hline Total & 113 & $100 \%$ \\
\hline
\end{tabular}

No que se refere ao tipo de artigo, foi possível verificar que na ReA/UFSM no período de 2008 a 2011 grande parte dos artigos publicados são empíricos.

\subsubsection{Abordagem de pesquisa}

A Tabela 6 evidencia as publicações quanto à abordagem de pesquisa.

Tabela 6 - Classificação dos artigos quanto à abordagem de pesquisa

\begin{tabular}{lll}
\hline Abordagem de pesquisa & $\mathbf{N}$ & $\mathbf{\%}$ \\
\hline Qualitativa & 66 & $58 \%$ \\
\hline Quantitativa & 36 & $32 \%$ \\
\hline Qualitativa e Quantitativa & 11 & $10 \%$ \\
\hline Total & $\mathbf{1 1 3}$ & $\mathbf{1 0 0 \%}$ \\
\hline
\end{tabular}

Verifica-se a predominância de pesquisas de natureza qualitativa (aproximadamente $60 \%$ )nas publicações da ReA/UFSM, considerando o período analisado. Esta característica demonstra que as publicações na Revista buscam estudos de um fenômeno sobre o qual ainda não se tem ideias bem definidas.

\subsubsection{Método de pesquisa}

A Tabela 7 mostra a classificação dos artigos quanto ao método de pesquisa.

Tabela 7 - Classificação dos artigos quanto ao método de pesquisa

\begin{tabular}{lll}
\hline Método de Pesquisa & $\mathbf{N}$ & $\mathbf{\%}$ \\
\hline Base qualitativa & 70 & $62 \%$ \\
\hline Survey & 37 & $33 \%$ \\
\hline Bibliometria & 6 & $5 \%$ \\
\hline Total & $\mathbf{1 1 3}$ & $\mathbf{1 0 0 \%}$ \\
\hline
\end{tabular}

A utilização de métodos de base qualitativa predominou nas publicações analisadas, o que vai ao encontro do fato da maioria das publicações utilizarem a abordagem qualitativa. 
O segundo método mais utilizado nos estudos foi o survey, seguido da bibliometria. Não foram identificados estudos utilizando o método experimento.

\subsubsection{Natureza de pesquisa}

A Tabela 8 evidencia que quanto à natureza de pesquisa, os artigos analisados são em sua maioria de natureza exploratória.

Tabela 8 - Classificação dos artigos quanto à natureza de pesquisa

\begin{tabular}{lll}
\hline Natureza da Pesquisa & $\mathbf{N}$ & $\mathbf{\%}$ \\
\hline Exploratória & 47 & $42 \%$ \\
\hline Descritiva & 44 & $39 \%$ \\
\hline Exploratória e Descritiva & 21 & $19 \%$ \\
\hline Causal & 1 & $1 \%$ \\
\hline Total & $\mathbf{1 1 3}$ & $\mathbf{1 0 0 \%}$ \\
\hline
\end{tabular}

A partir da análise das publicações evidenciou-se que em média são publicados 28 artigos por ano na revista. As instituições que obtiveram um maior número de publicações na ReA/ UFSM foram a Universidade de São Paulo, a Universidade Federal de Santa Maria e a Universidade Federal de Minas Gerais. Predominam as publicações nas áreas de Administração de Empresas, Administração de Recursos Humanos e Mercadologia. Os artigos analisados são em sua maioria empíricos, adotam uma abordagem qualitativa, utilizam métodos de base qualitativa e quanto a natureza classificam-se como exploratórios.

A seguir procede-se a análise de acordo com o método utilizado: survey, base qualitativa e bibliométrico. De acordo com as particularidades de cada método, foram destacados os seguintes aspectos: natureza da pesquisa, embasamento conceitual, coleta e análise dos dados e limitações e recomendações.

4.4 Análise dos artigos que utilizam método do tipo survey

A Tabela 9 apresenta uma síntese dos resultados da análise dos 37 artigos que utilizam o método survey.

Tabela 9 - Síntese da análise dos artigos baseados em pesquisa do tipo survey

\begin{tabular}{|c|c|c|c|c|}
\hline & Artigos do tipo Survey & & $\mathbf{N}$ & $\%$ \\
\hline \multirow{4}{*}{ Classificação } & \multirow{4}{*}{ Natureza da Pesquisa } & Exploratória & 7 & $19 \%$ \\
\hline & & Descritiva & 27 & $73 \%$ \\
\hline & & Causal & 1 & $3 \%$ \\
\hline & & Exploratória e Descritiva & 2 & $5 \%$ \\
\hline \multirow{7}{*}{ Embasamento conceitual } & \multirow{3}{*}{ Teorias e conceitos de base } & Aprofundado & 16 & $43 \%$ \\
\hline & & Superficial & 21 & $57 \%$ \\
\hline & & Ausente & 0 & $0 \%$ \\
\hline & \multirow{2}{*}{ Objetivos } & Sim & 37 & $100 \%$ \\
\hline & & Não & 0 & $0 \%$ \\
\hline & \multirow{2}{*}{$\begin{array}{l}\text { Questão de pesquisa, hipóte- } \\
\text { ses ou pressuposto de base }\end{array}$} & Sim & 24 & $65 \%$ \\
\hline & & Não & 13 & $35 \%$ \\
\hline
\end{tabular}




\begin{tabular}{|c|c|c|c|c|}
\hline & Artigos do tipo Survey & & $\mathbf{N}$ & $\%$ \\
\hline \multirow{5}{*}{ Desenho da pesquisa } & \multirow{3}{*}{ Modelo de Pesquisa } & Sim, com clareza & 18 & $49 \%$ \\
\hline & & Sim, com pouca clareza & 10 & $27 \%$ \\
\hline & & Não & 9 & $24 \%$ \\
\hline & \multirow{2}{*}{$\begin{array}{l}\text { Operacionalização das variá- } \\
\text { veis }\end{array}$} & Sim & 17 & $46 \%$ \\
\hline & & Não & 20 & $54 \%$ \\
\hline \multirow{10}{*}{ Coleta dos dados } & \multirow{3}{*}{ Tipo de dados } & Primários & 20 & $54 \%$ \\
\hline & & Secundários & 11 & $30 \%$ \\
\hline & & Primários e secundários & 6 & $16 \%$ \\
\hline & \multirow{4}{*}{ Forma de coleta de dados } & Entrevista pessoal & 23 & $62 \%$ \\
\hline & & Eletrônico & 3 & $8 \%$ \\
\hline & & Telefone & 1 & $3 \%$ \\
\hline & & Outro & 10 & $27 \%$ \\
\hline & \multirow{3}{*}{ Tipo de amostra } & Probabilística & 9 & $24 \%$ \\
\hline & & Não probabilística & 8 & $22 \%$ \\
\hline & & Não declarado & 20 & $54 \%$ \\
\hline \multirow{10}{*}{ Análise dos dados } & \multirow{3}{*}{ Confiabilidade } & Ausente & 24 & $65 \%$ \\
\hline & & Cronbach & 10 & $27 \%$ \\
\hline & & Outro & 3 & $8 \%$ \\
\hline & \multirow{3}{*}{ Validade dos construtos } & Ausente & 28 & $76 \%$ \\
\hline & & $\begin{array}{l}\text { Convergente/Discrimi- } \\
\text { nante }\end{array}$ & 2 & $5 \%$ \\
\hline & & Outro & 7 & $19 \%$ \\
\hline & \multirow{4}{*}{ Tipo de análise* } & Univariada & 20 & $54 \%$ \\
\hline & & Bivariada & 1 & $3 \%$ \\
\hline & & Multivariada & 19 & $51 \%$ \\
\hline & & Outros & 1 & $3 \%$ \\
\hline \multirow{5}{*}{ Limitações e Recomendações } & \multirow{2}{*}{$\begin{array}{l}\text { Apresenta as limitações do } \\
\text { estudo }\end{array}$} & Sim & 17 & $46 \%$ \\
\hline & & Não & 20 & $54 \%$ \\
\hline & \multirow{3}{*}{$\begin{array}{l}\text { Apresenta recomendações } \\
\text { para pesquisas futuras }\end{array}$} & Sim & 22 & $59 \%$ \\
\hline & & Não & 15 & $41 \%$ \\
\hline & & Total & 37 & $100 \%$ \\
\hline
\end{tabular}

*A soma destas categorias é maior que $100 \%$ pois é possível a utilização de mais de um tipo de análise por artigo

No que se refere às publicações que utilizam o método survey evidenciou-se que a maioria dos artigos: possui natureza descritiva, apresenta teoria e conceitos de base superficiais, apresenta questão de pesquisa, hipóteses ou pressuposto de base, traz o modelo de pesquisa com clareza, porém não apresenta a operacionalização das variáveis. Em relação à coleta dos dados foi possível verificar que são utilizados predominantemente dados primários, coletados em sua maioria por entrevista pessoal e a maior parte das publicações não declararam o tipo de amostra utilizada.

Quanto à análise dos dados, a confiabilidade e a validade dos construtos estão ausentes na maioria dos estudos e predomina o tipo de análise univariada. Na apresentação dos resultados, constatou-se que mais da metade dos artigos não apresenta limitações em relação ao estudo realizado, porém a maioria traz recomendações para futuros estudos. 
4.5 Análise dos artigos baseados em pesquisas qualitativas

A Tabela 10 contém a síntese da análise dos artigos baseados em pesquisas qualitativas. Entre os anos de 2008 e 2011 foram publicados 70 artigos que utilizaram métodos de base qualitativa.

Tabela 10 - Síntese dos artigos baseados em pesquisas qualitativas

\begin{tabular}{|c|c|c|c|c|}
\hline & Artigos de Base Qualitativa & & $\mathbf{N}$ & $\%$ \\
\hline \multirow{3}{*}{ Classificação } & \multirow{3}{*}{ Natureza da Pesquisa } & Exploratória & 40 & $57 \%$ \\
\hline & & Descritiva & 11 & $16 \%$ \\
\hline & & Exploratória e Descritiva & 19 & $27 \%$ \\
\hline \multirow{7}{*}{$\begin{array}{l}\text { Embasamento } \\
\text { conceitual }\end{array}$} & \multirow{3}{*}{ Teorias e conceitos de base } & Aprofundado & 33 & $47 \%$ \\
\hline & & Superficial & 35 & $50 \%$ \\
\hline & & Ausente & 2 & $3 \%$ \\
\hline & \multirow{2}{*}{ Objetivos } & Sim & 62 & $89 \%$ \\
\hline & & Não & 8 & $11 \%$ \\
\hline & \multirow{2}{*}{$\begin{array}{l}\text { Questão de pesquisa, hipóteses } \\
\text { ou pressuposto de base }\end{array}$} & Sim & 21 & $30 \%$ \\
\hline & & Não & 49 & $70 \%$ \\
\hline \multirow{8}{*}{$\begin{array}{l}\text { Desenho da pes- } \\
\text { quisa }\end{array}$} & \multirow{3}{*}{ Modelo de Pesquisa } & Sim, com clareza & 14 & $20 \%$ \\
\hline & & Sim, com pouca clareza & 9 & $13 \%$ \\
\hline & & Não & 47 & $67 \%$ \\
\hline & \multirow{5}{*}{ Tipo de Metodologia } & Etnografia & 1 & $1 \%$ \\
\hline & & Groundedtheory & 1 & $1 \%$ \\
\hline & & Estudo de caso & 40 & $57 \%$ \\
\hline & & Fenomenológico & 1 & $1 \%$ \\
\hline & & Outros & 27 & $39 \%$ \\
\hline \multirow{9}{*}{$\begin{array}{l}\text { Coleta dos da- } \\
\text { dos }\end{array}$} & \multirow{3}{*}{ Tipo de dados } & Primários & 23 & $33 \%$ \\
\hline & & Secundários & 26 & $37 \%$ \\
\hline & & Primários e secundários & 21 & $30 \%$ \\
\hline & \multirow{6}{*}{ Forma de coleta de dados* } & Pesquisa documental & 36 & $51 \%$ \\
\hline & & Entrevista não estruturada & 7 & $10 \%$ \\
\hline & & Entrevista estruturada & 7 & $10 \%$ \\
\hline & & Entrevista semi- estruturada & 21 & $30 \%$ \\
\hline & & Grupos de foco & 3 & $4 \%$ \\
\hline & & Outros & 18 & $26 \%$ \\
\hline \multirow{3}{*}{$\begin{array}{l}\text { Análise dos da- } \\
\text { dos }\end{array}$} & \multirow{3}{*}{ Tipo de análise** } & Análise de conteúdo & 25 & $36 \%$ \\
\hline & & Análise do discurso & 1 & $1 \%$ \\
\hline & & Outros & 44 & $63 \%$ \\
\hline \multirow{4}{*}{$\begin{array}{l}\text { Limitações e Re- } \\
\text { comendações }\end{array}$} & \multirow{2}{*}{$\begin{array}{l}\text { Apresenta as limitações do es- } \\
\text { tudo }\end{array}$} & Sim & 20 & $29 \%$ \\
\hline & & Não & 50 & $71 \%$ \\
\hline & \multirow{2}{*}{$\begin{array}{l}\text { Apresenta recomendações para } \\
\text { pesquisas futuras }\end{array}$} & Sim & 26 & $37 \%$ \\
\hline & & Não & 44 & $63 \%$ \\
\hline
\end{tabular}

*A soma destas categorias é maior que $100 \%$ pois é possível a utilização de mais de um tipo de coleta de dados por artigo.

**A soma destas categorias é maior que $100 \%$ pois é possível a utilização de mais de um tipo de análise por artigo.

Os dados referentes às publicações que utilizaram métodos de base qualitativa demonstram que predominam os artigos de natureza exploratória. Quanto ao embasamento conceitual as teorias e os conceitos de base de mais da metade dos artigos são superficiais ou ausentes. 
Também grande parte dos estudos apresenta objetivos definidos. Contudo, a maioria dos artigos não apresenta questões de pesquisa ou pressupostos de base. Grande parte das publicações não apresenta modelo de pesquisa com clareza e na maioria dos artigos o método adotado foi o estudo de caso. Quanto aos tipos de dados utilizados nas pesquisas, constatou-se que $37 \%$ dos artigos são baseados em dados secundários, $33 \%$ em dados primários e 30\% em dados primários e secundários. Em relação à coleta de dados, ainda que alguns artigos tenham utilizado mais de um tipo de coleta, predominam a pesquisa documental e a entrevista semi-estruturada nos artigos qualitativos analisados.

Para a análise dos dados, os autores recorreram em sua maioria a outros tipos de análise dos dados, seguido da análise de conteúdo.

Quanto às limitações e recomendações para estudos futuros, a maioria dos artigos não contém indicação de limitações e não apresentam recomendações para pesquisas futuras.

\subsection{Análise dos artigos bibliométricos}

Conforme apresentado na Tabela 11 a pesquisa identificou seis artigos bibliométricos publicados no período entre 2008 e 2011 na ReA/UFSM.

Tabela 11 - Síntese dos artigos bibliométricos

\begin{tabular}{|c|c|c|c|c|}
\hline & Artigos do tipo Bibliométrico & & $\mathbf{N}$ & $\%$ \\
\hline Classificação & Natureza da Pesquisa & Descritiva & 6 & $100 \%$ \\
\hline \multirow{6}{*}{$\begin{array}{l}\text { Embasamento } \\
\text { conceitual }\end{array}$} & \multirow{2}{*}{ Teorias e conceitos de base } & Aprofundado & 2 & $33 \%$ \\
\hline & & Superficial & 4 & $67 \%$ \\
\hline & \multirow{2}{*}{ Objetivos } & Sim & 6 & $100 \%$ \\
\hline & & Não & 0 & $0 \%$ \\
\hline & \multirow{2}{*}{$\begin{array}{l}\text { Questão de pesquisa, hipóteses } \\
\text { ou pressuposto de base }\end{array}$} & Sim & 1 & $17 \%$ \\
\hline & & Não & 5 & $83 \%$ \\
\hline \multirow{5}{*}{$\begin{array}{l}\text { Desenho da pes- } \\
\text { quisa }\end{array}$} & \multirow{3}{*}{ Modelo de Pesquisa } & Sim, com clareza & 4 & $67 \%$ \\
\hline & & Sim, com pouca clareza & 1 & $17 \%$ \\
\hline & & Não & 1 & $17 \%$ \\
\hline & \multirow{2}{*}{ Operacionalização das variáveis } & Sim & 2 & $33 \%$ \\
\hline & & Não & 4 & $67 \%$ \\
\hline \multirow{4}{*}{ Coleta dos dados } & \multirow{3}{*}{ Tipo de dados } & Primários & 0 & $0 \%$ \\
\hline & & Secundários & 6 & $100 \%$ \\
\hline & & Primários e secundários & 0 & $0 \%$ \\
\hline & Forma de coleta de dados & Pesquisa Bibliométrica & 6 & $100 \%$ \\
\hline Análise dos dados & Tipo de análise & Univariada & 6 & $100 \%$ \\
\hline \multirow{5}{*}{$\begin{array}{l}\text { Limitações e Re- } \\
\text { comendações }\end{array}$} & \multirow{2}{*}{$\begin{array}{l}\text { Apresenta as limitações do es- } \\
\text { tudo }\end{array}$} & Sim & 4 & $67 \%$ \\
\hline & & Não & 2 & $33 \%$ \\
\hline & \multirow{3}{*}{$\begin{array}{l}\text { Apresenta recomendações para } \\
\text { pesquisas futuras }\end{array}$} & Sim & 5 & $83 \%$ \\
\hline & & Não & 1 & $17 \%$ \\
\hline & & Total & 6 & $100 \%$ \\
\hline
\end{tabular}

No que se refere aos artigos bibliométricos constatou-se que as teorias e os conceitos de base são superficiais na maioria dos artigos e todos apresentaram objetivos bem definidos. Em grande parte das publicações não foram apresentadas questão de pesquisa, hipóteses ou pressupostos de base. Em relação ao desenho de pesquisa, a maioria dos artigos apresenta modelo de pesquisa com clareza, contudo não apresentam operacionalização de variáveis. A análise 
dos dados foi univariada na totalidade dos artigos bibliométricos analisados na presente pesquisa. A maior parte dos artigos apresenta limitações do estudo e indica recomendações para pesquisas futuras.

Assim, considerando o objetivo do presente artigo e as variáveis selecionadas para fins de análise, constatou-se que: (i) a maioria dos artigos publicados na ReA são empíricos; (ii) a Universidade de São Paulo (USP) e a Universidade Federal de Santa Maria são as instituições com o maior volume de artigos publicados; (iii) as publicações da ReA/UFSM enquadram-se predominantemente na área de Administração de Empresas, (iv) a abordagem da pesquisa é qualitativa na maioria dos artigos; (v) o método de pesquisa que prevalece na análise é de base qualitativa; e (vi)sobressai a pesquisa exploratória na natureza de pesquisa.

\section{CONSIDERAÇÕES FINAIS}

O presente estudo teve como objetivo analisar os artigos publicados na Revista de Administração - ReA/UFSM no período de 2008 a 2011, buscando identificar características gerais das publicações, bem como aspectos relacionados a base conceitual, aspectos metodológicos, coleta e análise dos dados, limitações e recomendações da produção científica do referido periódico.

A partir da análise dos artigos publicados na ReA/UFSM foi possível obter um panorama geral das publicações bem como uma análise detalhada de cada método de pesquisa utilizado nos estudos (survey, base qualitativa e bibliométrico).

De acordo com os resultados gerais, pode-se constatar que dos artigos analisados, grande parte são de base empírica, possuem abordagem qualitativa, método qualitativo e natureza exploratória. Com base nessas constatações, os resultados das pesquisas que utilizam o delineamento survey permitiram verificar que: em relação à natureza de pesquisa, a maioria dos artigos são de natureza descritiva, apresentam teoria e conceitos de base superficiais, mais da metade dos artigos não apresentaram questão de pesquisa, hipóteses ou pressuposto de base, $49 \%$ apresentam modelo de pesquisa com clareza e $54 \%$ não apresentam a operacionalização das variáveis. Em relação à coleta dos dados foi possível verificar que a maioria dos artigos utiliza dados primários, coletados por meio de entrevista pessoal, porém não declararam o tipo de amostra adotado. Além disso, observou-se que na análise dos dados a confiabilidade e a validade dos construtos estão ausentes na maioria dos estudos e evidenciou-se a predominância da análise univariada. Ressalta-se ainda que, com relação à apresentação dos resultados, a maioria dos artigos não indicou limitações do estudo realizado, contudo apresentaram recomendações para futuros estudos.

No que se refere aos resultados dos artigos que utilizaram métodos de base qualitativa, pode-se verificar que: predomina a natureza de pesquisa exploratória, a maioria dos estudos apresentam teoria e conceitos de base superficiais, mais da metade dos artigos não apresentaram questão de pesquisa, hipóteses ou pressuposto de base e modelo de pesquisa com clareza, sendo que o tipo de método predominante foi o estudo de caso. Em relação à coleta dos dados foi possível verificar que na metade dos artigos a coleta foi realizada por meio de entrevista pessoal. Cabe ressaltar que, com relação à apresentação dos resultados, constatou-se que mais da metade dos artigos não apresentaram limitações em relação ao estudo realizado, bem como recomendações para futuros estudos.

No que se refere aos artigos bibliométricos verificou-se que apresentam objetivos bem definidos e teorias superficiais na maioria dos artigos. Em relação ao desenho de pesquisa, a 
maioria dos artigos apresenta modelo de pesquisa com clareza. A análise dos dados foi univariada e a maior parte dos artigos apresenta limitações do estudo e indica recomendações para pesquisas futuras.

A análise da produção científica de um periódico da área de Administração que iniciou as suas atividades há cerca de cinco anos, torna-se relevante pelo fato de possibilitar traçar um panorama das publicações e da evolução do periódico, bem como estabelecer novas perspectivas e desafios a fim de garantir a consolidação e longevidade da Revista.

A partir da análise dos artigos publicados na Revista de Administração da UFSM - ReA/ UFSM, foi possível identificar os elementos de investigação científica presente nos trabalhos nos últimos quatro anos, permitindo traçar um perfil das publicações do periódico. A fim de que a ReA/UFSM se consolide cada vez mais no meio acadêmico de modo a garantir a sua longevidade, a partir do panorama das publicações dos últimos quatro anos sugere-se que a Revista estimule a publicação de estudos quantitativos, tendo em vista o paradigma funcionalista predominante atualmente nos estudos em Administração. E também sugere-se um maior incentivo para trabalhos oriundos de diferentes instituições, tendo em vista que a Universidade Federal de Santa Maria e a Universidade de São Paulo juntas detém 25\% do total de autorias das publicações do periódico no período de 2008 a 2012. 


\section{REFERÊNCIAS}

BERTERO, C. O. Meio século de RAE. Revista de Administração de Empresas, São Paulo, v.51, n.3, 2011, p. 224-226.

CHIAPPETA JABBOUR. Gestão ambiental empresarial: um levantamento da produção cientifica brasileira divulgada em periódicos da área de Administração entre 1996 e 2005. RAC

- Revista de Administração Contemporânea, v. 12, n.3, 2008, p.689.

CUNHA, L. Publicações científicas por meio eletrônico: critérios, cuidados, vantagens e desvantagens. Perspectivas em ciência da informação, Belo Horizonte, v. 2, n. 1, p. 77 92, 1997.

GONÇALVES. C. A.; MEIRELLES, A. M. Projetos e relatórios de pesquisa em administração. São Paulo: Atlas, 2004.

HOPPEN, N.; MOREAU, E.; LAPOINTE, L. Avaliação de artigos de pesquisa em sistemas de informação: proposta de um guia. In: ENCONTRO DA ASSOCIAÇÃO NACIONAL DE PÓS GRADUAÇÃO E PESQUISA EM ADMINISTRAÇÃO, 21, 1997, Rio das Pedras. Anais... Rio das Pedras: ANPAD, 1997.

JABBOUR, C. J. C.; SANTOS, F. C. A.; BARBIERI, J. C. Gestão ambiental empresarial: um levantamento da produção científica brasileira divulgada em periódicos da área de administração entre 1996 e 2005.RAC - Revista de Administração Contemporânea, Curitiba, v. 12, n. 3, 2008, 689-715.

PERIN, M. G.; SAMPAIO, C. H.; FROEMMING, L. M. S.; LUCE, F. B. A perspectiva survey em artigos de marketing nos ENANPADs da década de 90. In: ENCONTRO DA ASSOCIAÇÃO NACIONAL DE PÓS GRADUAÇÃO E PESQUISA EM ADMINISTRAÇÃO, 24, 2000, Florianópolis. Anais... Florianópolis: ANPAD, 2000.

PINTO, M. de R; LARA, J. D. O que se publica sobre comportamento do Consumidor no
Brasil, afinal? Revista de Administração da UFSM, Santa Maria, v. 01, n. 03, set./dez., 2008.

SERRA, F. A. R.; FIATES, G. G.; FERREIRA, M. P. Publicar é difícil ou faltam competências? RAM

- Revista De Administração Mackenzie, v. 9, n. 4, edição especial, 2008, p. 32-55.

SILVA, M. R. Análise bibliométrica da produção científica docente do programa de pós-graduação em educação especial/ UFSCar: 1998-2003. Dissertação (Mestrado em Educação Especial) - Universidade Federal de São Carlos, São Carlos, 2004.

WOOD JR., T.; CHUEKE, G. V. Ranking de produção científica em Administração de Empresas no Brasil. RAM -Revista de Administração Mackenzie, v. 9, n. 4, edição especial, 2008, p. 13-31. 
